\title{
STATE-SPACE MODELLING OF DATA ON MARKED INDIVIDUALS
}

Olivier GIMENEZ ${ }^{1,2,6}$, Vivien ROSSI ${ }^{3,4,5}$, Rémi CHOQUET ${ }^{1}$, Camille DEHAIS ${ }^{1}$, Blaise DORIS ${ }^{1,3}$, Hubert VARELLA ${ }^{1,3}$, Jean-Pierre VILA ${ }^{3}$ and Roger PRADEL ${ }^{1}$

${ }^{1}$ Centre d'Ecologie Fonctionnelle et Evolutive/CNRS - UMR 5175 1919 Route de Mende 34293 Montpellier - FRANCE

${ }^{2}$ Centre for Research into Ecological and Environmental Modelling University of St Andrews, St Andrews

The Observatory, Buchanan Gardens, KY16 9LZ - SCOTLAND

${ }^{3}$ Laboratoire d'Analyse des Systèmes et Biométrie - UMR 729 INRA/ENSAM

2 Place Pierre Viala, 34060 Montpellier - FRANCE

${ }^{4}$ Institut de Modélisation Mathématiques de Montpellier - UMR 5149 Universite Montpellier 2 CC051, Place Eugène Bataillon, 34095 Montpellier Cedex 5 - FRANCE ${ }^{5}$ Laboratoire de Biostatistique Institut Universitaire de Recherche Clinique 641 Avenue du Doyen Gaston Giraud, 34093 Montpellier Cedex 5 - FRANCE ${ }^{6}$ E-mail: olivier.gimenez@cefe.cnrs.fr

\footnotetext{
Abstract

State-space models have recently been proposed as a convenient and flexible framework for specifying stochastic models for the dynamics of wild animal populations. Here we focus on the modelling of data on marked individuals which is
} 
frequently used in order to estimate demographic parameters while accounting for imperfect detectability. We show how usual models to deal with capture-recapture and ring-recovery data can be fruitfully written as state-space models. An illustration is given using real data and a Bayesian approach using MCMC methods is implemented to estimate the parameters. We give important future developments facilitated by the SSM formulation. Overall, the general state-space modelling framework is shown to have a great potential in population ecology modelling.

\section{Introduction}

The estimation of animal survival is essential in population biology to investigate population dynamics, with important applications in the understanding of ecological, evolutionary, conservation and management issues for wild populations (Pollock, 1991; Williams et al., 2002). While the time to event is known in medical, social or engineering sciences (death, marriage and failure respectively), models for estimating wild animal survival must incorporate nuisance parameters to account for incomplete detectability in monitoring individuals (Schwarz et Seber, 1999). Typically, individuals are captured, marked and can be resighted or recaptured (encountered thereafter) to construct encounter histories which consist of sequences of 1's and 0's according to whether a detection occurs or not. The likelihood for such data arises from products of multinomial distributions whose cell probabilities are complex functions of survival probabilities - parameters of primary interest - and encounter probabilities - nuisance parameters (Cormack, 1964; Jolly, 1965; Seber, 1965 - CJS thereafter).

In this note, we show how the population process can be fruitfully disentangled, by distinguishing the underlying demographic process, i.e. the survival (as well as transitions between sites/states if needed), from its observation, i.e. the detectability. 
1 This leads us to consider a natural formulation for capture-recapture models using state2 space models (SSMs). Our contribution is in line with a recent paper by Buckland et 3 al. (2004) who have proposed to adopt SSMs as a convenient and flexible framework 4 for specifying stochastic models for the dynamics of wild animal populations.

Thus far, SSMs have been mainly used for time series of animal counts (de Valpine, 6 2004; Millar and Meyer, 2000) or animal locations (Anderson-Sprecher and Ledolter, 7 1991) to allow true but unobservable states (the population size or trajectory) to be 8 inferred from observed but noisy data (see Clark et al., 2005 and Wang, 2006 for

22 (e.g. Lebreton et al., 1992). 


\section{$1 \quad 2.1 \quad$ Likelihood}

2 We first define the observations and then the states of the system. We assume that $3 n$ individuals are involved in the study with $T$ encounter occasions. Let $X_{i, t}$ be the ${ }_{4}$ binary random variable taking values 1 if individual $i$ is alive at time $t$ and 0 if it is ${ }_{5}$ dead at time $t$. Let $Y_{i, t}$ be the binary random variable taking values 1 if individual $i$ is 6 encountered at time $t$ and 0 otherwise. Note that we consider the encounter event as 7 being physically captured or barely observed. The parameters involved in the likelihood 8 are $\phi_{i, t}$, the probability that an animal $i$ survives to time $t+1$ given that it is alive 9 at time $t(t=1, \ldots, T-1)$, and $p_{i, t}$ the probability of detecting individual $i$ at time $t$ ${ }_{10}(t=2, \ldots, T)$. Let finally $e_{i}$ be the occasion where individual $i$ is encountered for the 11 first time. A general state-space formulation of the CJS model is therefore given by:

$$
\begin{aligned}
Y_{i, t} \mid X_{i, t} & \sim \operatorname{Bernoulli}\left(X_{i, t} p_{i, t}\right), \quad t>e_{i} \\
X_{i, t+1} \mid X_{i, t} & \sim \operatorname{Bernoulli}\left(X_{i, t} \phi_{i, t}\right), \quad t \geq e_{i}
\end{aligned}
$$

where Equation (1) and Equation (2) are the observation and the state equations respectively. This formulation naturally separates the nuisance parameters (the encounter probabilities) from the parameters of actual interest i.e. the survival probabilities, the latter being involved exclusively in the state Equation (2). Such a clear distinction between a demographic process and its observation makes the description of a biological dynamic system much simpler and allows complex models to be fitted (Pradel, 2005; Clark et al., 2005). We will refer to this formulation as the individual state-space CJS model (individual SSM CJS hereafter). The rationale behind the above formulation is as follows. We give the full details for the observation Equation (1) only, as a similar reasoning easily leads to Equation (2). If individual $i$ is alive at time $t$, then it has prob- 
$2 Y_{i, t}$ is distributed as Bernoulli $\left(p_{i, t}\right)$ given $X_{i, t}=1$. Now if individual $i$ is dead at time $t$,

4 given $X_{i, t}=0$. Putting together those two pieces of reasoning, the distribution of the

5 observation $Y_{i, t}$ conditional on the state $X_{i, t}$ is given by Equation (1).

ability $p_{i, t}$ of being encountered and probability $1-p_{i, t}$ otherwise, which translates into then it cannot be encountered, which translates into $Y_{i, t}$ is distributed as Bernoulli(0) Statistical inference then requires the likelihood of the state-space model specified above. Assuming independence of individuals, the likelihood is given by the product of all individual likelihood components. The likelihood component for individual $i$ is the probability of the vector of observations $\mathbf{Y}_{i}^{T}=\left(Y_{i, e_{i}}, \ldots, Y_{i, T}\right)$ which gathers the information set up to time $T$ for this particular individual. The likelihood component corresponding to individual $i$ is therefore given by (e.g. Harvey, 1989)

(1)

$$
\int_{X_{i, 0}}, \ldots, \int_{X_{i, T}}\left\{\prod_{t=e_{i}+1}^{T}\left[Y_{i, t} \mid X_{i, t}\right]\left[X_{i, t} \mid X_{i, t-1}\right] d X_{i, t}\right\}\left[X_{i, e_{i}}\right] d X_{i, e_{i}}
$$

where $[X]$ denotes the distribution of $X$ and $X_{i, e_{i}}$ the initial state of individual $i$ which is assumed to be alive. Because we deal with binary random vectors, we used the counting measure instead of the Lebesgue measure.

In its original formulation, the CJS makes important assumptions regarding individuals. First, all individuals share the same parameters, which means that the survival and detection probabilities depend on the time index only. In mathematical notation, we have $\phi_{i t}=\phi_{t}$ and $p_{i t}=p_{t}$ for all $i=1, \ldots, n$, so Equation (1) and Equation (2) become $X_{i, t+1} \mid X_{i, t} \sim \operatorname{Bernoulli}\left(X_{i, t} \phi_{t}\right)$ and $Y_{i, t} \mid X_{i, t} \sim \operatorname{Bernoulli}\left(X_{i, t} p_{t}\right)$ respectively. Second, the CJS model also assumes independence between individuals. By using simple relationships between Bernoulli and Binomial distributions, one can finally fruitfully 
1 formulates the original CJS model under the following state-space model:

2

3

${ }_{4}$ where $X_{t}^{+}$is the number of survivors of marked individuals from time $t-1, X_{t}^{-}$, plus the

$$
\begin{array}{r}
Y_{t} \mid X_{t}^{-} \sim \operatorname{Bin}\left(X_{t}^{-}, p_{t}\right) \\
X_{t+1}^{-} \mid X_{t}^{+} \sim \operatorname{Bin}\left(X_{t}^{+}, \phi_{t}\right)
\end{array}
$$
newly marked individuals at time $t$, and $Y_{t}$ is the total number of marked individuals encountered at time $t$. We will refer to this formulation as the population state-space CJS model (population SSM CJS hereafter). Interestingly, specifying the system under a state-space formulation now requires much less equations than the individual SSM CJS model, which may avoid the computational burden. Nevertheless, while the individual SSM CJS involves parameters for every single individual and sampling occasion, the population SSM CJS model makes the strong assumptions that all individuals behave the same as well as independently, which may be of little relevance from the biological point of view. To cope with this issue, in-between modelling can be achieved by considering age effects or groups classes in the population SSM model (Lebreton et al., 1992). Finally, covariates can be incorporated in order to assess the effect of environment such as climate change, most conveniently by writing the relationship between the target probabilities and the predictors on the logit scale (Pollock, 2002).

\subsection{Implementation}

Fitting capture-recapture models in a state-space framework is complicated due to the high-dimensional integral involved in the individual likelihood Equation (3). To circumvent this issue, several techniques have been proposed including Kalman filtering, Monte-Carlo particle filtering (such as sequential importance sampling) and MCMC (see 
Clark et al., 2005 and Wang, 2006 for reviews). Our objective here is not to discuss 2 these different methods. For our purpose, we adopt the MCMC technique which is now widely used in biology (Ellison, 2004; Clark, 2005), in particular for estimating animal 4 survival (Seber et Schwarz, 1999; Brooks et al., 2000). Besides, this is to our knowledge 5 the only methodology which comes with an efficient and flexible program to implement 6 it, which, in our case, will allow biologists to easily and rapidly adopt our approach.

\section{$7 \quad 2.3$ Illustration}

8 We consider capture-recapture data on the European dipper (Cinclus cinclus) that were 9 collected between 1981 and 1987 (Lebreton et al., 1992). The data consists of marking and recaptures of 294 birds ringed as adults in eastern France. We applied standard maximum-likelihood estimation (Lebreton et al. 1992) and MCMC techniques (Brooks et al. 2000) using the product-multinomial likelihood and the state-space likelihood of

Equation (3) in combination with Equation (1) and Equation (2). We ran two overdispersed parallel MCMC chains to check whether convergence was reached (Gelman, 1996). We used 10,000 iterations with 5,000 burned iterations for posterior summarization. We used uniform flat priors for both survival and detection probabilities. The simulations were performed using WinBUGS (Spiegelhalter et al., 2003). The R (Ihaka and Gentleman, 1996) package R2WinBUGS (Sturtz et al., 2005) was used to call WinBUGS and export results in R. This was especially helpful when converting the raw encounter histories into the appropriate format, generating initial values and calculate posterior modes. The programs are available in Appendix A. Posterior summaries for encounter and survival probabilities are given in Table 1, along with their posterior probability distributions that are displayed in Figure 1.

[Table 1 about here.] 
Survival estimates were uniformally similar whatever the method used (Table 1). In particular, there is a clear decrease in survival 1982-1983 and 1983-1984, corresponding to a major flood during the breeding season in 1983 (Lebreton et al., 1992).

In contrast, posterior medians of detection probabilities using the CJS SSM approach are quite different from the classical maximum likelihood estimates, but more similar to the posterior medians obtained with the product-multinomial likelihood approach (Table 1). These discrepancies are no longer present when posterior modes are examined, as expected (recall that we use non-informative uniform distributions as priors for all parameters).

The last survival probability as well as the last detection probability are estimated with high variability (Table 1 and Figure 1). The fact that these two parameters cannot be separately estimated is not surprising since the CJS model is known to be parameter-redundant (Catchpole and Morgan, 1997). Also, the first survival probability and the first detection probability are weakly identifiable, due to the fact that very few individuals were marked at the first sampling occasion (approximately $7 \%$ of the full data set).

\section{$3 \quad$ Further state-space modelling}

\subsection{Multistate capture-recapture models}

Multistate capture-recapture models (Arnason, 1973; Schwarz et al., 1993; AS hereafter) are a natural generalization of the CJS model in that individuals can move between states, according to probabilities of transition between those states. States can be either geographical sites or states of categorical variables like reproductive status or size class 
1 (Lebreton and Pradel, 2002). We provide here a state-space modelling formulation 2 of the AS model (Dupuis, 1995; Newman, 1998; Clark et al., 2005). Without loss of 3 generality, we consider 2 states. Let $X_{i, t}$ be the random state vector taking values ${ }_{4}(1,0,0),(0,1,0)$ and $(0,0,1)$ if, at time $t$, individual $i$ is alive in state 1,2 or dead ${ }_{5}$ respectively. Let $Y_{i, t}$ be the random observation vector taking values $(1,0,0),(0,1,0)$ 6 and $(0,0,1)$ if, at time $t$, individual $i$ is encountered in state 1,2 or not encountered. 7 Parameters involved in the modelling include $\phi_{i, t}^{r s}$, the probability that an animal $i$ 8 survives to time $t+1$ given that it is alive at time $t(t=1, \ldots, T-1)$ and makes the 9 transition between state $r$ and state $s$ over the same interval $(r, s=1,2)$, as well as $p_{i, t}^{r}$ the probability of detecting individual $i$ at time $t$ in state $r(t=2, \ldots, T, r=1,2)$. A state-space formulation for the multistate AS model is given by:

13

$$
\begin{array}{r}
Y_{i, t} \mid X_{i, t} \sim \text { Multinomial }\left(1, X_{i, t}\left[\begin{array}{ccc}
p_{i, t}^{1} & 0 & 1-p_{i, t}^{1} \\
0 & p_{i, t}^{2} & 1-p_{i, t}^{2} \\
0 & 0 & 1
\end{array}\right]\right) \\
X_{i, t+1} \mid X_{i, t} \sim \text { Multinomial } \\
\left(1, X_{i, t}\left[\begin{array}{ccc}
\phi_{i, t}^{11} & \phi_{i, t}^{12} & 1-\phi_{i, t}^{11}-\phi_{i, t}^{12} \\
\phi_{i, t}^{21} & \phi_{i, t}^{22} & 1-\phi_{i, t}^{21}-\phi_{i, t}^{22} \\
0 & 0 & 1
\end{array}\right]\right)
\end{array}
$$

where Equation (6) and Equation (7) are the observation and the state equations respectively. This formulation has similarities with that of Pradel (2005) who used hidden-Markov models to extend multistate models to cope with uncertainty in state assignment. Again, it should be noted that the state-space formulation allows the demographic parameters to be distinguished from the nuisance parameters. A similar reasoning to that adopted for the CJS model leads to Equations (6) and (7). As expected, Equation (6) and Equation (7) reduce to Equation (1) and Equation (2) if one 
considers a single state. Making similar assumptions as in the CJS model leads to the 2 population AS SSM.

\subsection{Ring-recovery models}

${ }_{4}$ The capture-recapture models presented above deals with apparent survival, which 5 turns out to be true survival if emigration is negligeable. When marks of individuals 6 (or individuals themselves) are actually recovered, true survival probabilities can be 7 estimated using ring-recovery models (Brownie et al., 1985; RR models hereafter). Let ${ }_{8} X_{i, t}$ be the binary random variable taking values 1 if individual $i$ is alive at time $t$ and 90 if it is dead at time $t$. Let $Y_{i, t}$ be the binary random variable taking values 1 if mark of individual $i$ is recovered at time $t$ and 0 otherwise. The parameters involved in the 11 likelihood are $\phi_{i, t}$, the probability that an animal $i$ survives to time $t+1$ given that 
1 is in a given state (dead or alive) at time $t$ and remains in this state till time $t+1$, then

2 its mark cannot be recovered, which translates into $Y_{i, t}$ is distributed as Bernoulli(0) 3 given $X_{i, t-1}=0$ and $X_{i, t}=0$ or $X_{i, t-1}=1$ and $X_{i, t}=1$ i.e. $X_{i, t-1}-X_{i, t}=0$. The dis4 tribution of the observation $Y_{i, t}$ conditional on the combination of states $X_{i, t-1}-X_{i, t}$ is 5 thus given by Equation (8). Similar comments to that of previous sections can be made 6 here as well. Finally, we note that because the probability distribution of the current observation does not only depend on the current state variable, the model defined by 8 Equation (8) and Equation (9) does not exactly matches the definition of a state-space 9 model but can be rewritten as such (see Appendix B).

10

\section{Discussion}

We have shown that, by separating the demographic process from its observation, CR models for estimating survival can be expressed as SSMs. In particular, the SSM formulation of the CJS model competes well with the standard method when applied to a real data set. Bearing this in mind, we see at least two further promising developments to our approach.

First, by scaling down from the population to the individual level while modelling the survival probabilities, random effects can readily be incorporated to cope with heterogeneity in the detection probability (Huggins, 2001) and deal with a frailty in the survival probability (Vaupel and Yashin, 1985). Second, the combination of various sources of information which has recently received a growing interest, (e.g. recovery and recapture data, Catchpole et al., 1998; recovery and census data, Besbeas et al., 2002; Besbeas et al., 2003) can now be operated/conducted in a unique SSM framework and hence benefits from the corpus of related methods. Of particular importance, we 
1 are currently investigating the robust detection of density-dependence by accounting

2 for error in the measurement of population size using the combination of census data 3 and data on marked individuals.

$4 \quad$ Because most often, data collected in population dynamics studies give only a noisy 5 output of the demographic process under investigation, the SSM framework provides 6 a flexible and integrated framework for fitting a wide range of models which, with 7 widespread adoption, has the potential to advance significantly ecological statistics 8 (Buckland et al., 2004; Thomas et al., 2005). 


\section{Acknowledgments}

2 O. Gimenez's research was supported by a Marie-Curie Intra-European Fellowship 3 within the Sixth European Community Framework Programme. This project was

4 funded by the Action Incitative Régionale BioSTIC-LR 'Modélisation intégrée en dy5 namique des populations : applications à la gestion et à la conservation'.

\section{${ }_{6} 6$ Literature Cited}

7 Anderson-Sprecher, R., Ledolter, J., 1991. State-space analysis of wildlife telemetry 8 data. Journal of the American Statistical Association. 86, 596-602.

9 Arnason, A.N., 1973. The estimation of population size, migration rates and survival 10 in a stratified population. Research in Population Ecology. 15, 1-8.

11 Besbeas, P., Freeman, S.N., Morgan, B.J.T., Catchpole, E.A., 2002. Integrating mark12 recapture-recovery and census data to estimate animal abundance and demographic parameters. Biometrics. 58, 540-547. Besbeas, P., Lebreton, J.D., Morgan, B.J.T., 2003. The efficient integration of abundance and demographic data. Journal of the Royal Statistical Society Series C - Applied Statistics. 52, 95-102.

17 Brooks, S. P., Catchpole, E. A., Morgan, B. J. T., 2000. Bayesian animal survival estimation. Statistical Science. 15, 357-376.

21 Washington.

22 23 for the dynamics of wild animal populations. Ecological Modelling. 171, 157-175. 
1 Catchpole, E.A., Freeman, S.N., Morgan, B.J.T., Harris, M.P., 1998. Integrated recov2 ery/recapture data analysis. Biometrics. 54, 33-46.

3 Choquet, R., Reboulet, A.-M., Pradel, R., Gimenez, O., Lebreton, J.-D., 2004. M4 SURGE: new software specifically designed for multistate capture-recapture models. 5 Animal Biodiversity and Conservation. 27, 207-215.

6 Clark, J.S., 2005. Why environmental scientists are becoming Bayesians. Ecology 7 Letters. 8, 2-14.

8 Clark, J.S., Ferraz, G.A., Oguge, N., Hays, H., DiCostanzo, J., 2005. Hierarchical Bayes 9 for structured, variable populations: From recapture data to life-history prediction. 10 Ecology. 86, 2232-2244.

11 Cormack, R. M., 1964. Estimates of survival from the sighting of marked animals. 12 Biometrika. 51, 429-438. 
2 of Computational and Graphical Statistics. 5, 299-314.

3 Jolly, G. M., 1965. Explicit estimates from capture-recapture data with both death and 4 immigration-stochastic model. Biometrika. 52, 225-247.

5 Lebreton, J.-D., Burnham, K. P., Clobert, J., Anderson, D. R., 1992. Modeling survival 6 and testing biological hypotheses using marked animals: A unified approach with case 7 studies. Ecological Monograph. 62, 67-118.

8 Lebreton, J.D., Almeras, T., Pradel, R., 1999. Competing events, mixtures of informa9 tion and multistrata recapture models. Bird Study. 46, 39-46.

Lebreton, J.D., Pradel, R., 2002. Multistate recapture models: modelling incomplete individual histories. Journal of Applied Statistics. 29, 353-369.

Millar, R.B., Meyer, R., 2000. Non-linear state space modelling of fisheries biomass dynamics by using Metropolis-Hastings within-Gibbs sampling. Journal of the Royal Statistical Society Series C - Applied Statistics. 49, 327-342.

Newman, K. B. 1998. State-space modeling of animal movement and mortality with application to salmon. Biometrics. 54, 1290-1314.

Pollock, K.H., 1991. Modeling capture, recapture, and removal statistics for estimation of demographic parameters for fish and wildlife populations - past, present, and future.

Journal of the American Statistical Association. 86, 225-238.

Pollock, K. H., 2002. The use of auxiliary variables in capture-recapture modelling: an overview. Journal of Applied Statistics. 29, 85-102.

Pradel, R., 2005. Multievent: An extension of multistate capture-recapture models to uncertain states. Biometrics. 61, 442-447.

Schwarz, C.J., Schweigert, J.F., Arnason, A.N., 1993. Estimating migration rates using tag-recovery data. Biometrics. 49, 177-193. 
1 Schwarz, C. J., Seber, G. A. F., 1999. Estimating animal abundance: review III.

2 Statistical Science. 14, 427-56.

3 Seber, G. A. F., 1965. A note on the multiple-recapture census. Biometrika. 52, $4 \quad 249-259$.

5 Spiegelhalter, D. J., Thomas, A., Best, N. G., Lunn, D., 2003. WinBUGS user manual 6 - Version 1.4. MRC Biostatistics Unit, Cambridge, UK.

7 Sturtz, S., Ligges, U., Gelman, A., 2005. R2WinBUGS: a package for running Win8 BUGS from R. Journal of Statistical Software. 12, 1-16.

9 Thomas, L., Buckland, S.T., Newman, K.B., Harwood, J., 2005. A unified framework 10 for modelling wildlife population dynamics. Australian and New Zealand Journal of 11 Statistics. 47, 19-34.

12 Vaupel, J.W., Yashin, A.I., 1985. Heterogeneity ruses - some surprising effects of 13 selection on population dynamics. American Statistician. 39, 176-185.

14 Wang, G., 2006. On the latent state estimation of nonlinear population dynamics using 15 Bayesian and non-Bayesian state-space models. Ecological Modelling. doi: 10.1016/ 16 j.ecolmodel.2006.09.004.

17 Williams, B. K., Nichols, J. D., Conroy, M. J., 2002. Analysis and management of 18 animal populations. Academic Press, San Diego.

\section{${ }_{19}$ Appendix A: WinBUGS code for fitting the CJS}

\section{model using the SSM formulation}

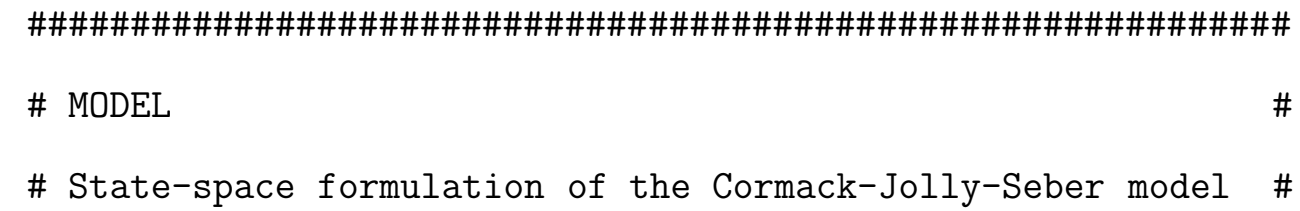




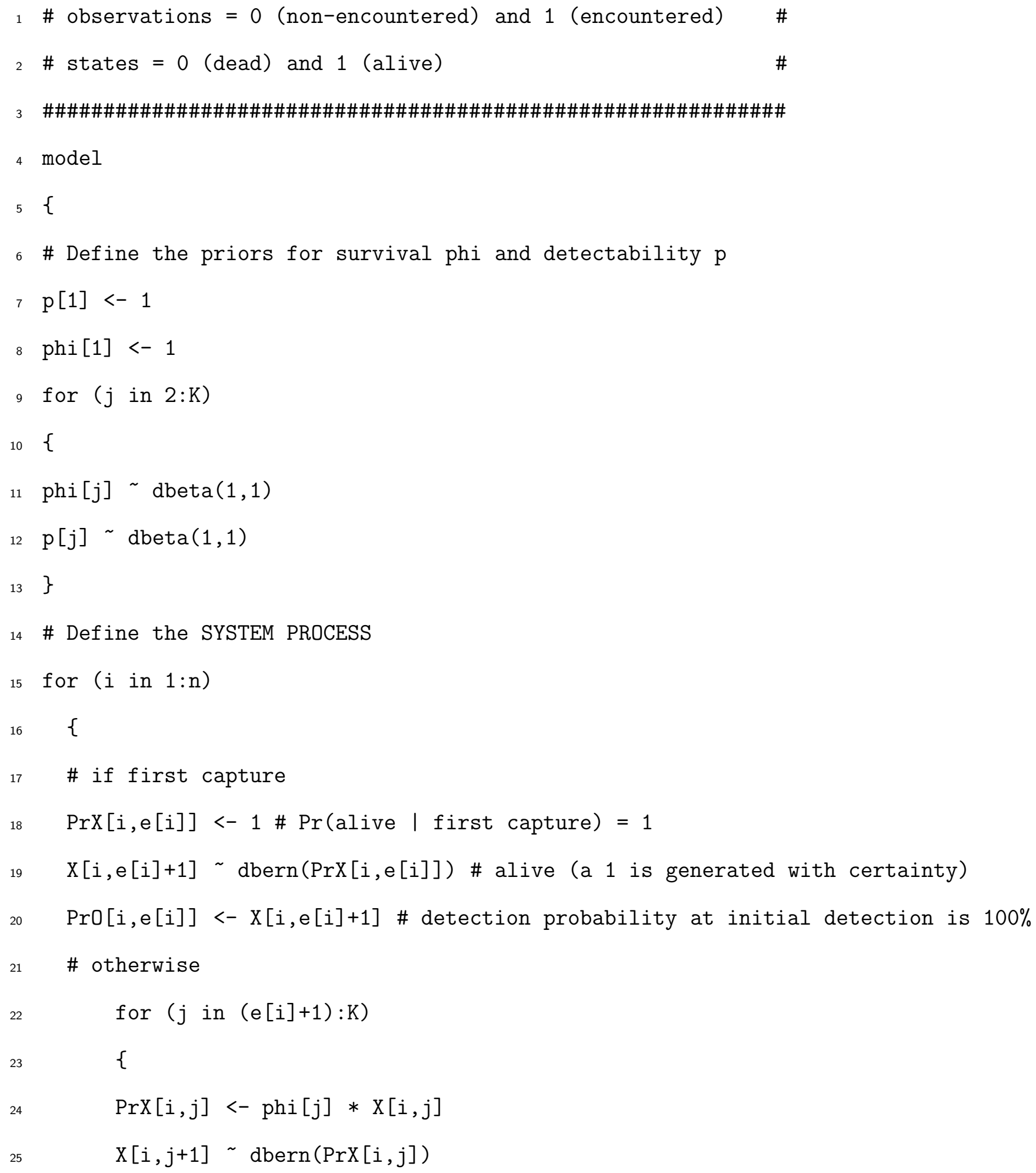




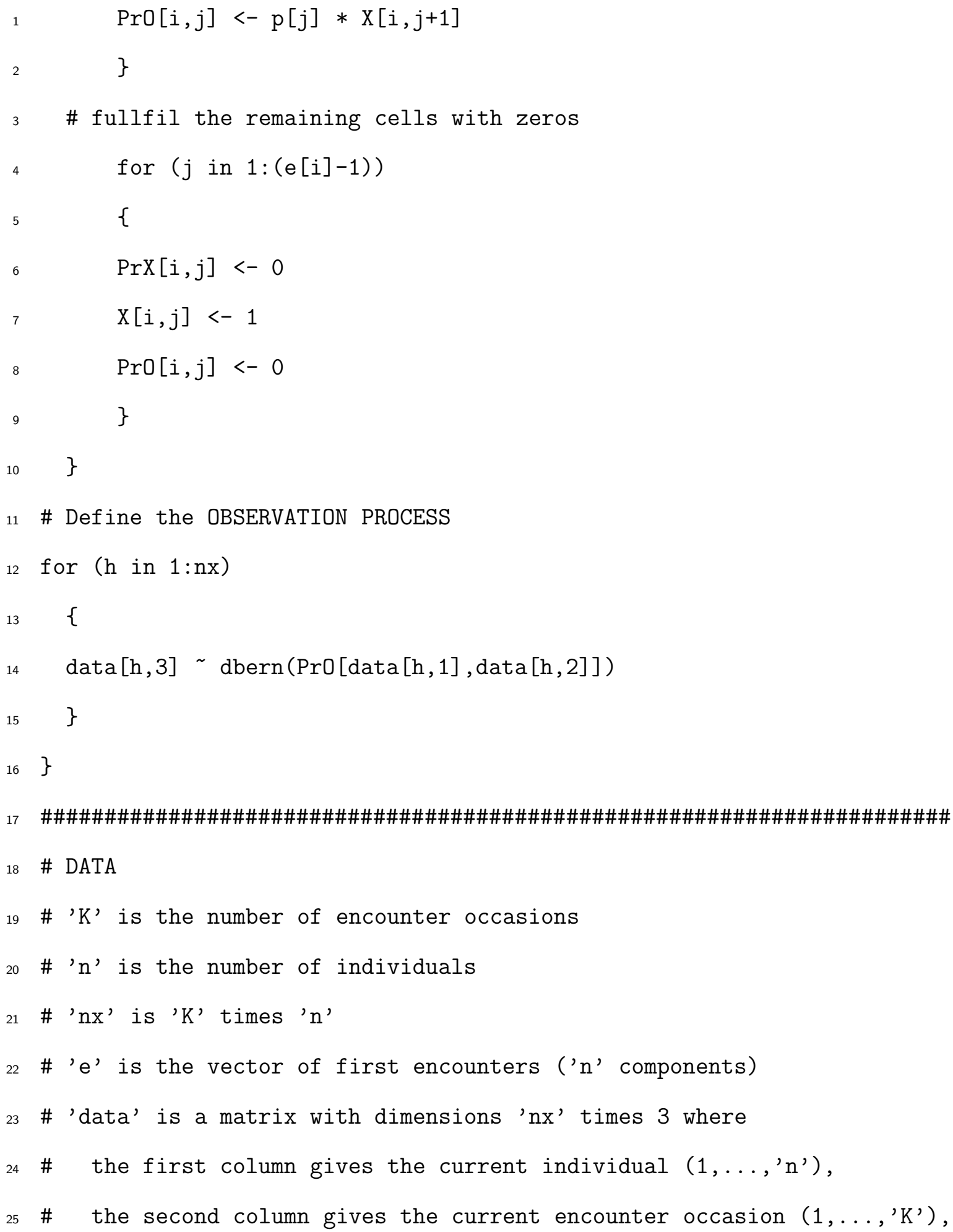


1 \# the third column gives the observation (= 1 if detection, = 0 otherwise)

2 \# corresponding to the current individual and current encounter occasion

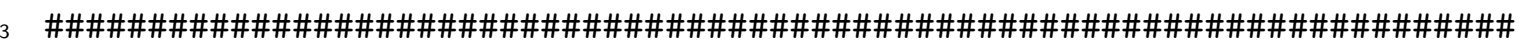

\section{${ }_{4}$ Appendix B}

${ }_{5}$ Let $Z_{i, t}=\left[X_{i, t-1}, X_{i, t}\right]$ be a bivariate random vector where its two components are

6 denoted $Z_{i, t}^{1}$ and $Z_{i, t}^{2}$. Equation (8) becomes

7

$$
Y_{i, t} \mid Z_{i, t} \sim \operatorname{Bernoulli}\left(\left(Z_{i, t}^{1}-Z_{i, t}^{2}\right) \lambda_{i, t}\right)
$$

8 and Equation (9) becomes

$$
Z_{i, t+1} \mid Z_{i, t}=\left\{\begin{array}{l}
\left(Z_{i, t+1}^{1} \mid Z_{i, t}\right)=Z_{i, t}^{2} \\
Z_{i, t+1}^{2} \mid Z_{i, t} \sim \operatorname{Bernoulli}\left(Z_{i, t}^{2} \phi_{i, t}\right)
\end{array}\right.
$$

10 The system defined by Equation (10) and Equation (11) is a state-space model and it 11 is equivalent to the model defined by Equation (8) and Equation (9).

12 Note that an alternative state-space formulation can be adopted using a multistate 13 formulation of the RR model (Lebreton et al., 1999) and Section 3.1. 


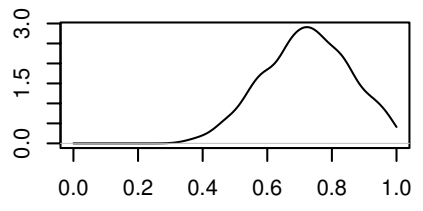

$\phi 1$

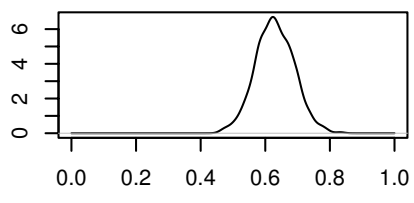

$\phi 4$

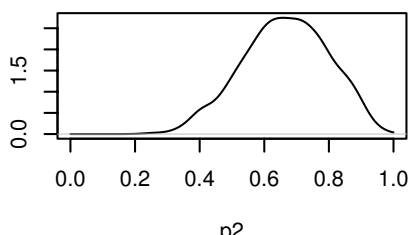

p2

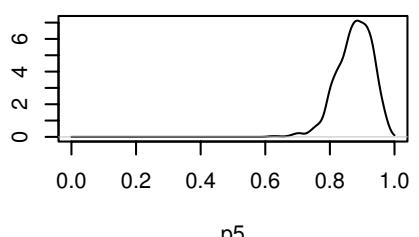

p5

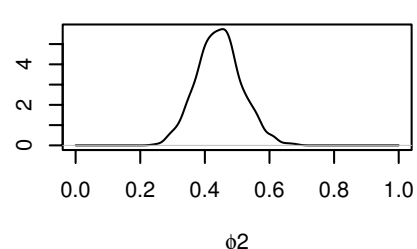

$\phi 2$

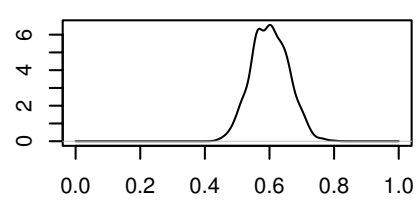

$\phi 5$

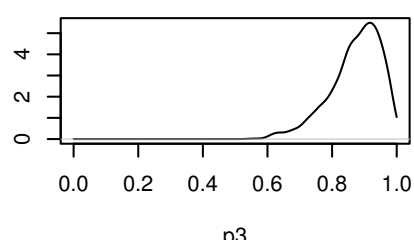

p3

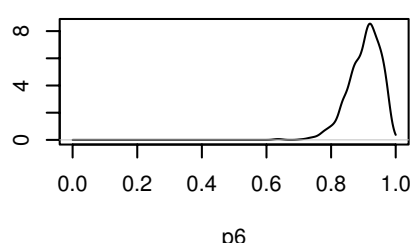

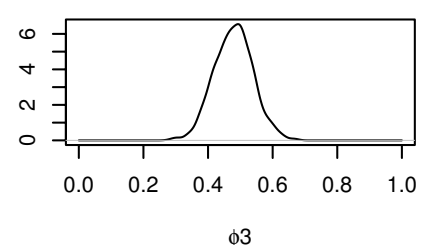

$\phi 3$
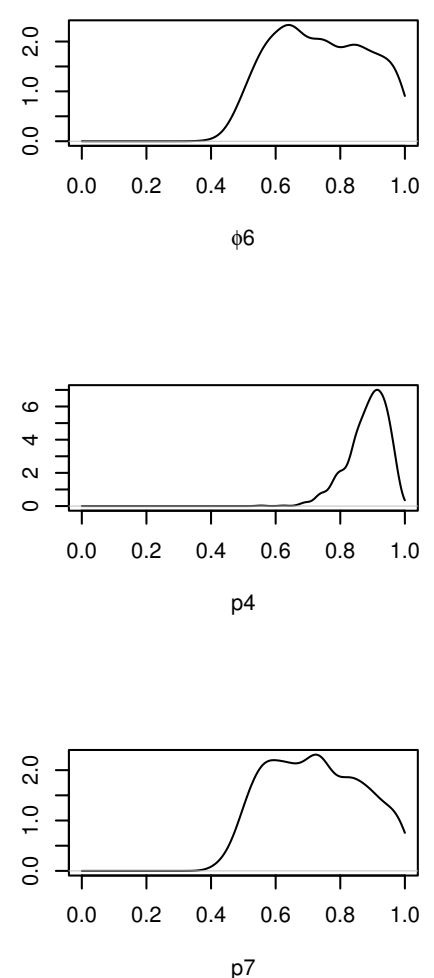

Figure 1: Posterior distributions for the survival and detection probabilities for the Dipper data using the CJS model applied to the Dipper data set as estimated by the state-space model and MCMC methods. 
Table 1: Estimated survival and detection probabilities for the Dipper data using the CJS model and three different methods, the state-space model (SSM) using a Monte Carlo Markov Chain (MCMC) method, the product-multinomial model (PMM) using a MCMC method and the PMM using a maximum-likelihood (ML) method. The two first methods were implemented using program WinBUGS (Spiegelhalter et al., 2003), while program M-SURGE (Choquet et al., 2004) was used to implement the last one.

\begin{tabular}{cccc}
\hline & SSM using MCMC & PMM using MCMC & PMM using ML \\
Parameter & Posterior median/mode (SD) & Posterior median/mode (SD) & MLE (SE) \\
\hline$\phi_{1}$ & $0.721 / 0.722(0.132)$ & $0.723 / 0.693(0.132)$ & $0.718(0.156)$ \\
$\phi_{2}$ & $0.448 / 0.456(0.071)$ & $0.448 / 0.460(0.071)$ & $0.435(0.069)$ \\
$\phi_{3}$ & $0.480 / 0.493(0.060)$ & $0.480 / 0.476(0.061)$ & $0.478(0.060)$ \\
$\phi_{4}$ & $0.628 / 0.624(0.061)$ & $0.627 / 0.616(0.060)$ & $0.626(0.059)$ \\
$\phi_{5}$ & $0.602 / 0.601(0.057)$ & $0.602 / 0.607(0.057)$ & $0.599(0.056)$ \\
$\phi_{6}$ & $0.713 / 0.640(0.142)$ & $0.720 / 0.628(0.143)$ & $-(-)^{*}$ \\
$p_{2}$ & $0.671 / 0.658(0.134)$ & $0.670 / 0.691(0.134)$ & $0.696(0.166)$ \\
$p_{3}$ & $0.883 / 0.918(0.083)$ & $0.883 / 0.904(0.083)$ & $0.923(0.073)$ \\
$p_{4}$ & $0.888 / 0.914(0.063)$ & $0.889 / 0.912(0.063)$ & $0.913(0.058)$ \\
$p_{5}$ & $0.882 / 0.885(0.057)$ & $0.883 / 0.904(0.057)$ & $0.901(0.054)$ \\
$p_{6}$ & $0.913 / 0.920(0.052)$ & $0.912 / 0.935(0.051)$ & $0.932(0.046)$ \\
$p_{7}$ & $0.735 / 0.724(0.142)$ & $0.727 / 0.648(0.143)$ & $-(-)^{*}$ \\
\hline
\end{tabular}

\footnotetext{
${ }^{*}$ Non-identifiability detected.
} 